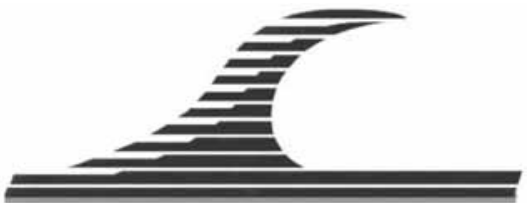

Revue Paralia, Volume 1 (2008) pp 1.1-1.14

Mots-clés : Vélocimétrie Doppler Ultrasonore (VDU),

Porosité, Interface, Sédiment, Nombre de Reynolds,

Mousse alvéolaire, Contrainte de frottement pariétale.

\title{
Étude de la répartition des vitesses interstitielles au sein d'un lit perméable sous un écoulement à surface libre
}

\section{Mustapha Kamel MIHOUBI ${ }^{1}$, Michel BELORGEY ${ }^{2}$, Daniel LEVACHER ${ }^{2}$, Ahmed KETTAB ${ }^{3}$}

${ }^{1}$ Ecole Nationale Supérieure de l’Hydraulique (ENSH), BP 31 Blida (09000), Algérie. mihkam@yahoo.fr

${ }^{2}$ Université de Caen, Faculté des sciences UMR 6143 CNRS-M2C, 24 rue des Tilleuls, 14000 Caen, France.

michel.belorgey@unicaen.fr; daniel.levacher@unicaen.fr

3 Laboratoire de Recherche Sciences de l'Eau (LRS), Ecole Nationale Polytechnique (ENP), 10 avenue Hassen Badi El Harrach, Alger 16200, Algérie. kettab@yahoo.fr

\section{Résumé:}

Les processus physiques associés au transport sédimentaire n’ont été abordés jusqu'à maintenant qu'à partir d'analyses du champ de vitesses au sein d'une veine fluide. La plupart des modèles ont été établis pour des écoulements sur des fonds imperméables. L'objectif de cette étude a été d'exploiter la technique ultrasonore VDU pour l'analyse du champ de vitesses au sein d'un écoulement permanent sur un fond sédimentaire poreux. Les vitesses sont mesurées pour différents lits sédimentaires, et ce, dans la veine fluide et au sein du sédiment. Les résultats acquis mettent en évidence :

- une répartition exponentielle des vitesses à l'intérieur du sédiment,

- une discontinuité de vitesse à l'interface eau-sédiment, entre la vitesse dans l'écoulement libre et la vitesse au sein du sédiment.

Ces résultats suggèrent la nécessité d'une nouvelle approche d'analyse du transport sédimentaire qui doit tenir compte de la réalité des processus physiques qui régissent la phase d’interface eau/sédiment.

Soumis le 4 février 2008, accepté le 9 juin 2008, en ligne le 16 juin 2008. La seule version examinée est celle écrite en français. La ou les autres versions n'étant pas examinées par le comité de rédaction de la revue, sont donc publiées sous l'entière responsabilité du ou des auteurs.

A TRANSLATED VERSION IN ENGLISH IS AVAILABLE ONLINE

Pour citer cet article :

MIHOUBI M.K., BELORGEY M., LEVACHER D., KETTAB A. (2008). Étude de la répartition des vitesses interstitielles au sein d'un lit perméable sous un écoulement à surface libre. Revue Paralia, $\mathrm{n}^{\circ}$ 1, pp 1.1-1.14.

DOI: 10.5150/revue-paralia.2008.001

(disponible en ligne - http://www.paralia.fr) 


\section{Introduction}

Le phénomène de transport sédimentaire est très complexe et les processus physiques qui lui sont associés sont encore mal connus.

En effet, faute d'une instrumentation appropriée, la modélisation de ce phénomène est encore basée sur deux types d’études indépendantes mais exploitées comme complémentaires, à savoir :

- L'analyse du champ des vitesses dans l'écoulement libre.

- La mesure des vitesses interstitielles au sein de l'écoulement en milieu poreux uniforme à gradient hydraulique constant.

La grande majorité des études qui ont conduit à la modélisation du champ des vitesses dans l'écoulement libre ont été réalisées pour un fond imperméable avec l'hypothèse de vitesse nulle au fond du lit. La contrainte de frottement à la paroi, indispensable à la modélisation du transport sédimentaire, étant déterminée soit à l'aide du gradient de vitesse à la paroi, soit à l'aide de la loi logarithmique de Prandtl et Von-Karman (GRAF \& ALTINAKAR, 1995). Les études dans ce domaine sont d'ailleurs très nombreuses.

Les études concernant la mesure des vitesses interstitielles au sein d'un milieu poreux, à gradient hydraulique constant, ont pour référence les travaux de Darcy basés sur une formulation linéaire et ceux de Forchheimer qui a présenté une relation non linéaire dans le cas de vitesses plus importantes. Par exemple, pour les sables grossiers et les graviers, la relation s'écrit :

$\mathrm{i}=\mathrm{a}_{0} * u+\mathrm{b}_{0} * \mathrm{u}^{2}$

où :

$i=$ gradient hydraulique moyen

$a_{o}$ et $b_{o}$ sont des coefficients qui caractérisent la structure du milieu poreux, ils dépendent de la porosité et de la tortuosité.

Dans ce contexte, il convient de citer les travaux de WARD (1964), AHMED et SUNADA (1969) qui proposent des modèles basés sur le nombre Reynolds des pores. Plus récemment, WAHYUDI et al. (2002) ont déterminé les coefficients du polynôme d'ordre 2 issu de loi de Forchheimer, équation (1), pour des sables homogènes de porosité " $n$ " variant entre 0,32 et 0,38 . De même on peut citer les travaux de MONTILLET (1995) qui exprime le gradient hydraulique à partir de la vitesse d'écoulement dans les pores, en tenant compte de la porosité et de la tortuosité du milieu poreux.

En fonction de la taille des pores dans le milieu, on peut dire qu'il existe deux domaines de porosité, une porosité capillaire pour un diamètre des pores inferieur à $2 \mathrm{~mm}$ et une porosité effective pour un milieu à macroporosité de diamètre supérieur à 2 mm (COMITI \& RENAUD, 1989). Par ailleurs, WAHYUDI (1998), 
Étude de la répartition des vitesses interstitielles au sein d'un lit perméable sous un écoulement à surface libre : 1.3

donne une classification aux écoulements en milieu poreux en fonction du diamètre des particules et de la vitesse d'infiltration.

Les études relatives aux problèmes d'interface entre milieu poreux et veine fluide sous écoulement à surface libre sont très restreintes. Les premiers fondements ont été donnés par SHIMIZU et al. (1990) (in KLAR, 2005) qui a présenté une formulation de type exponentielle pour un écoulement en milieu très perméable, en considérant que la vitesse est fonction de la vitesse d'infiltration et de glissement issue du changement d'interface.

Or, ces études ne correspondent pas à la réalité du processus physique lié au champ des vitesses généré par un écoulement à surface libre, au sein d'un fond sédimentaire, et ceci pour les raisons suivantes :

- Du fait de l'existence d'une vitesse interstitielle à l'interface eau-sédiment, la vitesse du fluide dans l'écoulement libre n'est pas nulle à la paroi.

- La répartition des vitesses au sein du sédiment n'est pas uniforme et il existe un gradient de vitesse en fonction de la profondeur.

Afin de mieux comprendre les processus physiques associés à ce phénomène, nous avons adapté la technique de vélocimétrie Doppler ultrasonore (VDU) à la mesure instantanée des vitesses interstitielles locales au sein d'un milieu poreux. Cette technique de mesure relativement récente, a connu un grand succès dans l'étude des écoulements opaques et chargés où d'autres méthodes, telles que la vélocimétrie laser, étaient inopérantes (LHERMITTE, 1983; WILLEMETZ, 1990). Elle a été déjà exploitée au laboratoire dans le cas d'écoulements à variation de température (MICHAUX-LEBLOND et al., 1996). Les travaux de CARPENTIER (2006), relatifs aux mesures des vitesses au sein du soubassement d'une digue soumise à l'action de la houle, qui de plus à validé la méthode par des tests expérimentaux de type Darcy dans le cas de mousses alvéolaires et à bulles. Les résultats présentés ici correspondent au cas d'un écoulement à surface libre sur un fond sédimentaire reconstitué. Les mesures de vitesse de l'écoulement interstitiel ont permis de préciser le profil de vitesse de l'écoulement intéressant la partie fluide mais aussi la partie poreuse du lit.

\section{Technique de mesures par VDU}

Le dispositif de mesures utilisé (vélocimètre Doppler ultrasonore, fonctionnant en mode pulsé : DOP 1000 (modèle 1032), permet grâce à l'utilisation de l'effet Doppler sur des ondes ultrasonore pulsées, de déterminer directement la vitesse interstitielle au niveau des pores du sol considéré.

Le principe est l'envoi d'une onde ultrasonore de fréquence connue $f_{0}$, qui à la rencontre d'une particule en mouvement dans le fluide renvoie une onde de 
fréquence décalée $f_{r}$ par effet Doppler. Ceci permet grâce à la différence de fréquence enregistrée $f_{D}=f_{0}-f_{r}$, de déterminer la mesure de la vitesse de la particule dans l'écoulement en ce point :

$u=\frac{c \cdot f_{D}}{2 f_{0} \cdot \cos \theta}$

$c=$ vitesse du son dans l'eau, valeur validée pour les mousses alvéolaires et à bulles conservées pour les billes de verre et le sable $\theta=$ angle du faisceau ultrasonore avec l'axe de l'écoulement

Si on ajoute à cette mesure de fréquence, la détermination du temps de parcours du train d'ondes en aller-retour on accède à la position de la particule. Ainsi, en mode pulsé, on peut accéder à la résolution "spatio-temporelle" souhaitée, c'est-àdire l'établissement du profil de vitesse sur une profondeur d'exploration adaptée le long du faisceau ultrasonore. Cependant, dans cette étude, nous nous sommes limités à une position définie (porte) au centre de la veine fluide.

\section{Dispositif expérimental et technique de traitement des mesures}

\subsection{Canal à flux continu}

Le dispositif est constitué d'un canal d'une longueur totale de 3,5 $\mathrm{m}$ et de section rectangulaire de $(0,10 \mathrm{~m} \times 0,25 \mathrm{~m})$, permettant d'effectuer des mesures de calibration de vitesse par le vélocimètre Doppler ultrasonore (figure 1).

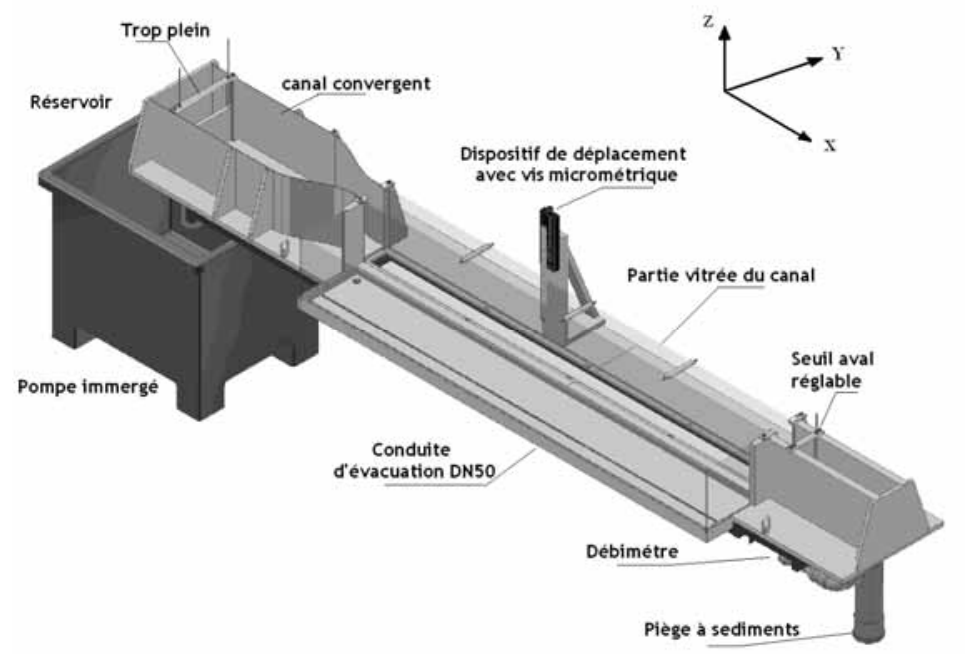

Figure 1. Description du canal à écoulement permanent à surface libre.

Les parois et le fond du canal sont vitrés d'une épaisseur de $10 \mathrm{~mm}$, permettant ainsi de faire des observations et des mesures optiques. Le canal est alimenté par un réservoir à charge constante en matériau PVC de section carrée de côté 0,30 m 
Étude de la répartition des vitesses interstitielles au sein d'un lit perméable sous un écoulement à surface libre : 1.5

et $0,25 \mathrm{~m}$ de hauteur. L'arrivée d'eau se fait par un système de crépine, l'eau est tranquillisée par des plaques de mousse d'environ $5 \mathrm{~cm}$ d'épaisseur. Dans le canal, le débit est contrôlé par un seuil réglable, le trop-plein d'eau est évacué vers le réservoir d'alimentation du canal. L'alimentation du canal est assurée par un circuit fermé au moyen d'une pompe de débit maximal égal à $(5,0 \pm 0,01) \mathrm{l} / \mathrm{s}$. Pour une meilleure linéarisation de l'écoulement et afin de réduire les effets de turbulence, deux cages en nid d'abeilles ont été installées avant la sortie du convergent.

\subsection{Principe de mesure de vitesse}

La sonde est un transducteur piézoélectrique de fréquence d'émission $4 \mathrm{MHz}$, et de diamètre $12 \mathrm{~mm}$. Elle est placée sur un système de déplacement de $24,0 \mathrm{~mm}$ de largeur, $400 \mathrm{~mm}$ de longueur et 2,7 $\mathrm{mm}$ d'épaisseur. L'axe du faisceau ultrasonore est horizontal et fait un angle de $28^{\circ}$ avec l'axe du canal. Le déplacement vertical de la sonde est assuré au moyen d'une vis différentielle micrométrique, ce qui permet d'obtenir des micro-déplacements et un maximum de points de mesures dans le lit sédimentaire et à l'interface fluide-sédiment. Le déplacement minimal assuré par le dispositif de la sonde est de $0,5 \mathrm{~mm}$. Compte tenu des dimensions du faisceau ultrasonore, les mesures sont traitées par un modèle de filtration statistique. L'ensemble, transducteur et support de déplacement sont protégés par une plaque de faible courbure (figure 2), formant une colonne à sonde. Dans ce système préconisé nous avons tenu compte de la réfraction entre les milieux : eau-PVC, PVC-eau.

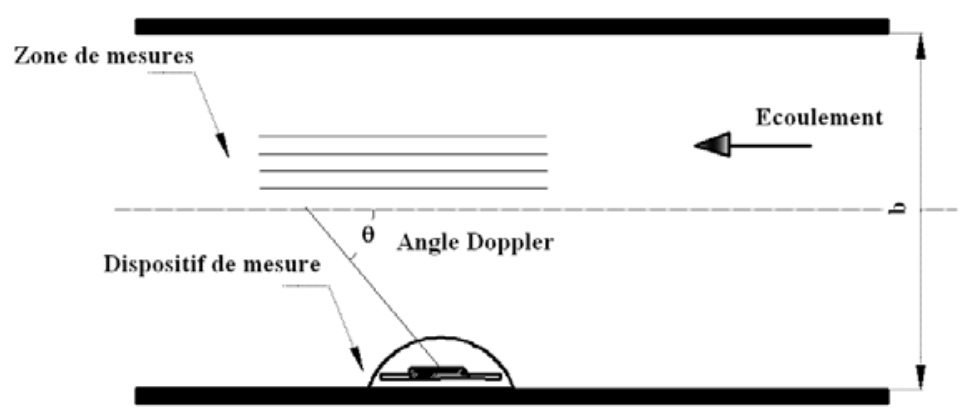

Figure 2. Vue en plan du système de mesures dans le canal.

Nous avons matérialisé le lit sédimentaire par des matériaux de porosité et de structure différentes : mousses alvéolaires et à bulles, billes de verre et du sable. L'épaisseur du lit poreux varie entre $35 \mathrm{~mm}$ et $75 \mathrm{~mm}$.

La plaque de protection du dispositif de déplacement est circulaire de diamètre 49,0 mm et d'épaisseur 2,0 mm et l'empattement dans le canal est égal à 8,0 mm. 


\section{$\underline{3.3}$ Traitement des mesures}

Le faisceau ultrasonore délivré par la sonde est sensiblement cylindrique (légèrement conique après une certaine distance). Dans notre cas, il était placé dans un plan horizontal perpendiculaire au plan médian de l'écoulement. Les particules soumises à ce faisceau émettent en réponse un écho lui aussi capté par la sonde. Cet écho qui dépend de l'angle entre le faisceau ultrasonore et la direction de l'écoulement possède deux caractéristiques principales :

- Sa fréquence qui permet d’accéder à la vitesse des particules (effet Doppler).

- Son intensité qui dépend de la taille des particules.

Le traitement du signal émis par les particules est alors abordé de deux manières différentes et bien distinctes :

- Celle liée à la taille des particules.

- Celle liée à la dimension du faisceau ultrasonore.

Dans cette étude de la vitesse interstitielle au sein des sédiments, nous avons deux tailles bien distinctes de particules :

- Les particules sédimentaires très grosses.

- Les particules (très petites) en suspension qui circulent entre les pores.

De ce fait :

- En supprimant dans le signal reçu, les échos de grande intensité, nous éliminons les échos issus des grosses particules sédimentaires dont la vitesse par ailleurs est nulle (sauf à l'interface eau-sédiment où il peut exister un transport sédimentaire, mais ceci fera l'objet d'une prochaine étude spécifique). Nous ne conservons ainsi que les échos issus des particules en suspension dans le fluide (avec l'hypothèse classique qu'elles ont la même vitesse que celui-ci).

- Par ailleurs, au sein du faisceau ultrasonore, le volume de mesure est un cylindre dont le diamètre est celui du faisceau $(8 \mathrm{~mm})$ et la longueur $(0,74 \mathrm{~mm})$ la profondeur de la "porte" (distance à laquelle sont effectuées les mesures). Les mesures enregistrées sont donc celles qui sont associées aux particules qui traversent ce volume de mesure. Or, compte tenu de la nature du champ des vitesses et de la section du volume de mesure (disque circulaire, ou légèrement elliptique), les particules les plus rapides ou les plus lentes traversent les secteurs circulaires supérieur ou inférieur, qui sont de section faible. Ces particules sont donc peu nombreuses dans leur catégorie.

Une étude statistique des valeurs enregistrées confirme cette interprétation. Et la forme Gaussienne des résultats nous permet :

- D’éliminer les valeurs peu nombreuses trop grandes ou trop faibles.

- De conserver une valeur moyenne des vitesses les plus probables comme étant la mesure des vitesses au niveau de l'axe du faisceau ultrasonore. 
Étude de la répartition des vitesses interstitielles au sein d'un lit perméable sous un écoulement à surface libre : 1.7

La figure 3 traduit de façon explicite la distribution des valeurs mesurées suivant la loi normale. Dans le milieu poreux, les vitesses ont été déterminées pour des intervalles de probabilité variant de 70 à $75 \%$ par rapport à la valeur de la vitesse moyenne et l'écart type d'un volume de mesures. L'erreur absolue estimée sur la détermination de la vitesse est égale à $\pm 0,2 \mathrm{~mm} / \mathrm{s}$.

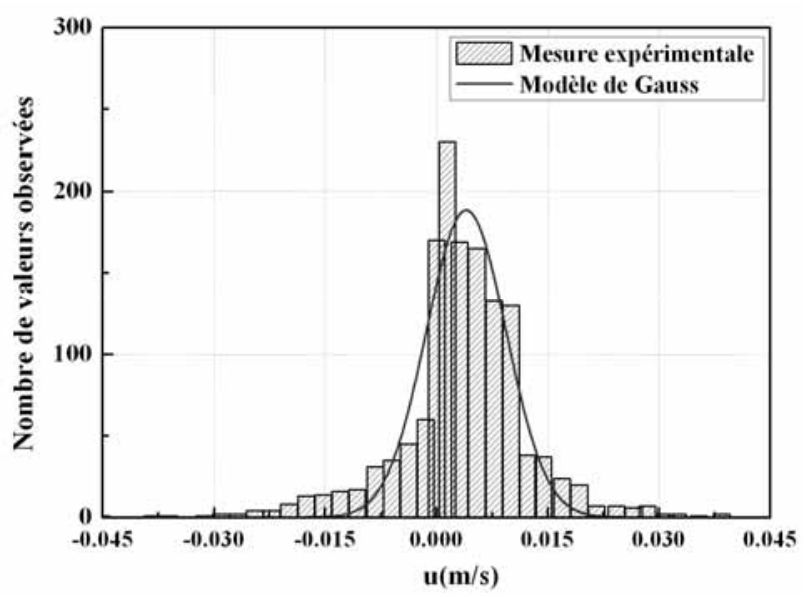

Figure 3. Distribution des vitesses comparée au modèle de Gauss pour un volume de mesures dans un lit en mousse alvéolaire $\left(Q_{f}=0,31 \mathrm{l} / \mathrm{s}, z=-3,0 \mathrm{~cm}\right)$.

\subsection{Validation des mesures}

Afin de comparer et valider les mesures obtenues par VDU, des mesures par vélocimétrie Doppler laser (VDL) ont été effectuées dans le cas d'un écoulement sur un lit imperméable à fond lisse. La figure 4, illustre bien les profils déterminés par les deux techniques.

A partir de l'ensemble des essais de mesures réalisés et validés par VDU suivant une porte fixe on peut donner en premier lieu une représentation de la vitesse instantanée d'un écoulement à surface libre sur un lit poreux en mousse alvéolaire pour trois positions verticales différentes de la sonde : au sein du lit, près de l'interface lit/fluide et dans la veine fluide (figure 5). Cette représentation illustre bien les gammes des vitesses suivant la hauteur $z$ ainsi que les effets de la turbulence générés en présence d'un écoulement hydrauliquement rugueux sur un lit perméable.

Le tableau 1 représente les caractéristiques physiques de l'étude et les paramètres de l'écoulement dans la veine fluide seule. En effet, le débit total est la somme des débits, dans la veine fluide et dans le sédiment, or ceux-ci sont difficilement quantifiables individuellement. De ce fait, nous avons privilégié les paramètres associés à la veine fluide seule pour caractériser l'écoulement et qui exploités par les hydrauliciens dans les études in situ. 


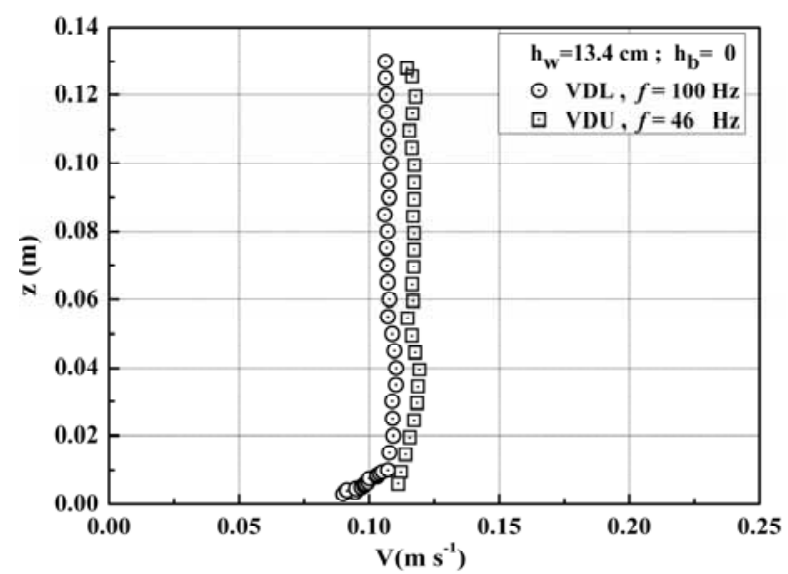

Figure 4. Profils comparatifs des vitesses obtenues par

les mesures de VDL et VDU pour $Q_{f}=1,47 \mathrm{l} / \mathrm{s}$.

Tableau 1. Caractéristiques des essais pour différents lits poreux.

\begin{tabular}{|c|c|c|c|c|c|c|c|c|c|}
\hline \multirow[t]{2}{*}{ Nature du lit } & $\boldsymbol{h}$ & $\frac{\boldsymbol{h}_{w}}{\boldsymbol{h}_{b}}$ & $u$ & $R e_{p}$ & $V$ & $\boldsymbol{R}_{h}$ & $Q f$ & $\operatorname{Re}$ & $\boldsymbol{F r}$ \\
\hline & $(\mathrm{cm})$ & --- & $(\mathrm{cm} / \mathrm{s})$ & --- & $(\mathrm{cm} / \mathrm{s})$ & $(\mathrm{cm})$ & $(l / s)$ & $x 10^{3}$ & --- \\
\hline \multirow[t]{4}{*}{ Mousse alvéolaire } & 8.00 & 0.23 & 0.061 & 52.52 & 7.30 & 1.15 & 0.11 & 0.84 & 0.19 \\
\hline & 11.70 & 0.80 & 0.103 & 86.11 & 5.90 & 2.55 & 0.31 & 1.41 & 0.08 \\
\hline & 12.50 & 0.92 & 0.124 & 106.8 & 22.80 & 2.73 & 1.37 & 5.84 & 0.30 \\
\hline & 12.75 & 0.96 & 0.384 & 330.3 & 31.60 & 2.78 & 1.98 & 8.25 & 0.40 \\
\hline \multirow[t]{3}{*}{ Mousse à bulles } & 10.90 & 0.45 & 0.020 & 4.95 & 7.10 & 2.02 & 0.24 & 1.35 & 0.12 \\
\hline & 11.50 & 0.53 & 0.076 & 12.43 & 14.60 & 2.22 & 0.58 & 3.04 & 0.23 \\
\hline & 12.90 & 0.72 & 0.170 & 19.90 & 27.60 & 2.60 & 1.50 & 6.74 & 0.38 \\
\hline \multirow[t]{3}{*}{ Billes } & 11.00 & 2.67 & 0.340 & 14.95 & 5.94 & 3.08 & 0.47 & 1.72 & 0.07 \\
\hline & 11.40 & 2.80 & 0.670 & 8.96 & 11.55 & 3.13 & 0.97 & 3.39 & 0.13 \\
\hline & 15.40 & 4.13 & 1.020 & 22.84 & 13.03 & 3.56 & 1.61 & 4.36 & 0.12 \\
\hline \multirow[t]{3}{*}{ Sable } & 11.70 & 2.90 & 0.430 & 0.81 & 5.70 & 3.18 & 0.50 & 1.70 & 0.06 \\
\hline & 14.70 & 3.90 & 0.850 & 1.55 & 11.50 & 3.50 & 1.35 & 3.78 & 0.11 \\
\hline & 15.40 & 4.13 & 1.840 & 3.35 & 13.00 & 3.56 & 1.61 & 4.35 & 0.12 \\
\hline
\end{tabular}

Paramètres fixés :

Lit en mousse alvéolaire $-h_{b}=6.5 \mathrm{~cm} ; D_{p}=3.1 \mathrm{~mm} ; n=96.4 \%$

Lit en mousse à bulles $-h_{b}=7.5 \mathrm{~cm} ; D_{p}=0.9 \mathrm{~mm} ; n=94.5 \%$

Lit en billes - $n=37.5 \% ; h_{b}=3 \mathrm{~cm} ; D=(3.8-4.4) \mathrm{mm}$

Lit en sable $-n=32.0 \% ; h_{b}=3 \mathrm{~cm} ; D_{50}=0.406 \mathrm{~mm}$ 
Étude de la répartition des vitesses interstitielles au sein d'un lit perméable sous un écoulement à surface libre: 1.9

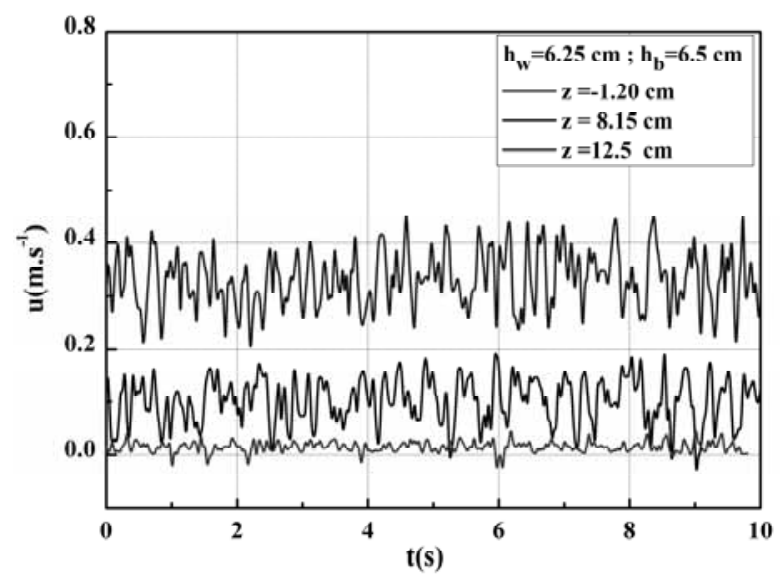

Figure 5. Evolution de la vitesse instantanée pour un écoulement sur un lit en mousse alvéolaire $\left(h=12,75 \mathrm{~cm}\right.$ et $\left.Q_{f}=1,98 \mathrm{l} / \mathrm{s}\right)$.

\section{Analyse des résultats expérimentaux}

Sur les figures 6(a) et 6(b) nous avons représenté les évolutions des vitesses obtenues par VDU pour plusieurs lits sédimentaires et différents débits d'écoulement en fonction de l'axe $z$ (profondeurs pour les sédiments et hauteur d'eau pour la veine fluide). La cote "0" correspond à l'interface eau/sédiment.

Comme le montre en détail la figure 7 (cas d'un lit en mousse alvéolaire), les résultats mettent nettement en évidence :

- Une forte discontinuité de vitesses à l'interface eau/sédiment, c'est-à-dire un gradient très important fonction du débit et de la nature du sédiment.

- Une vitesse non nulle sur le fond (pour la veine fluide).

- Une évolution exponentielle de la vitesse dans les pores des sédiments qui peut se mettre sous la forme :

$\mathrm{u}_{(z)}=\mathrm{A} \cdot \mathrm{e}^{\alpha \cdot z}$

$A$ et $\alpha$ sont deux paramètres expérimentaux qui dépendent des conditions d'écoulement en milieu poreux et à l'interface eau-sédiment.

Le paramètre caractérise la porosité du lit et le régime d'écoulement dans les pores du lit.

Pour mieux appréhender l'évolution du paramètre $\alpha$ suivant les caractéristiques du milieu poreux on utilise les grandeurs adimensionnelles suivantes :

$\mathrm{U}=\frac{\mathrm{U}}{\mathrm{u}_{\max }} \quad$ et $\quad Y=\frac{z}{h_{b}} \quad$ avec : $Y<0$

A partir du tracé des graphes $\ln (U)=\mathrm{f}(Y)$ qui correspondent aux mesures des vitesses interstitielles dans le lit poreux, on peut déduire la valeur du paramètre $\alpha$ à partir du coefficient directeur de la droite de régression linéaire. 


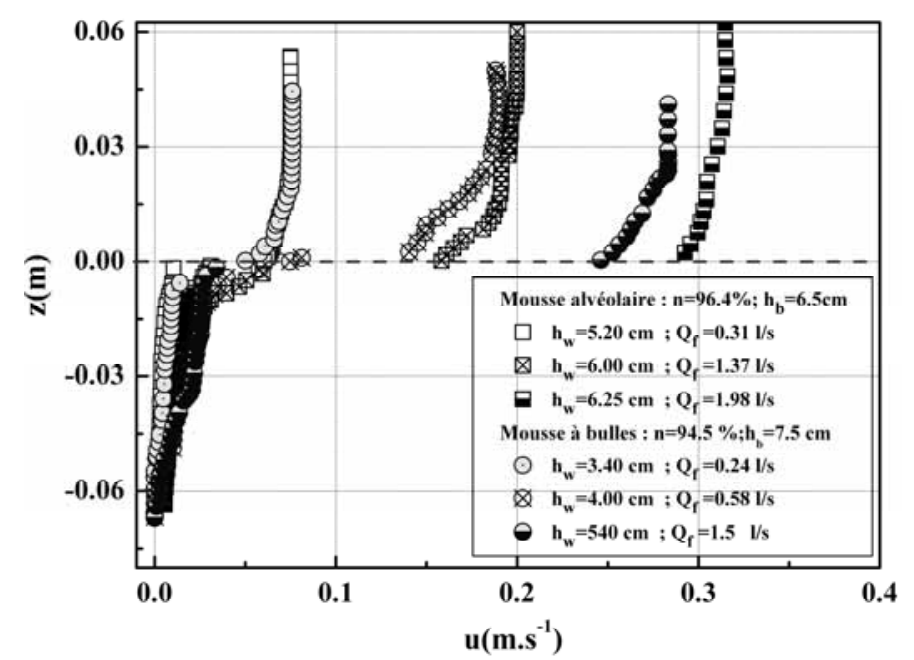

Figure 6(a). Profils de vitesses pour des lits poreux en mousse alvéolaire et en mousse à bulles.

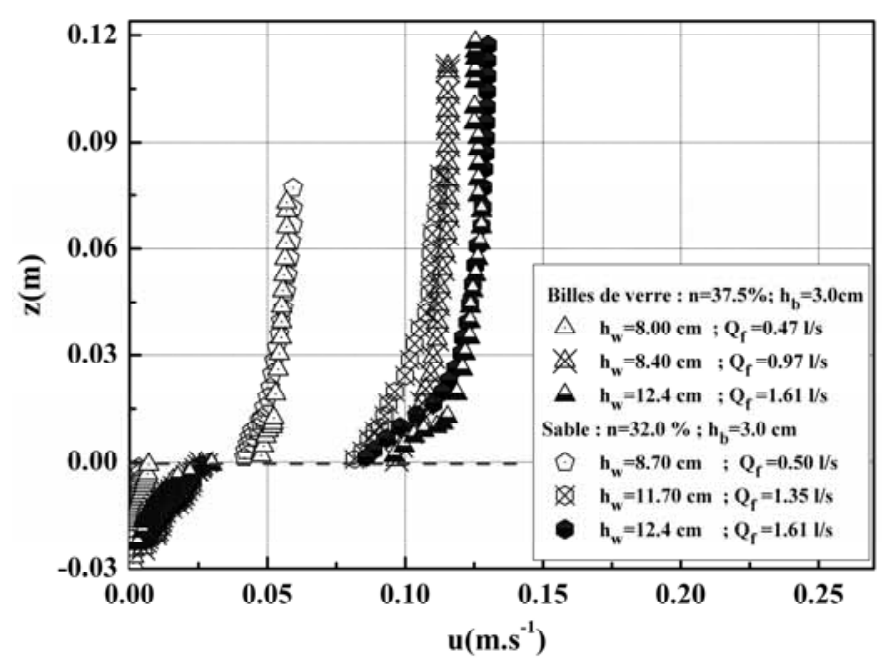

Figure 6(b). Profils de vitesses pour des lits poreux en billes de verre et en sable.

On constate que le paramètre dépend non seulement de la nature du sédiment mais aussi du régime de l'écoulement à l'échelle des pores autrement dit, il est influencé par la taille des pores du lit reconstitué (figure 8a et 8b).

L'examen de la variation du paramètre $\alpha$ en fonction du régime d'écoulement du milieu poreux en fonction du nombre de Reynolds des pores a permis de constater qu'il varie en fonction de la taille des pores (figure 9).

On remarque qu'il existe deux domaines distincts, le premier domaine caractérise les pores $D_{p}<2 \mathrm{~mm}$, le paramètre $\alpha$ est presque constant puis décroît pour des Reynolds $R_{e p}<10$. Par contre pour $D_{p}>2 \mathrm{~mm}$, le nombre de Reynolds augmente caractérisant ainsi le domaine d'écoulement interstitiel poreux turbulent. 
Étude de la répartition des vitesses interstitielles au sein d'un lit perméable sous un écoulement à surface libre : 1.11

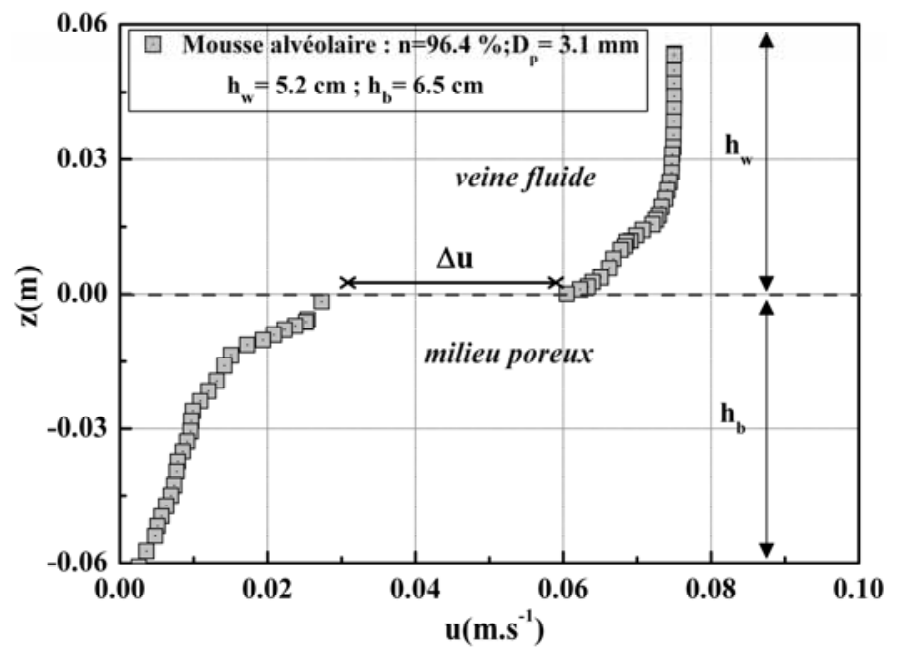

Figure 7. Détail de la discontinuité des vitesses à l'interface eau-sédiment $\left(h=11,7 \mathrm{~cm}\right.$ et $\left.Q_{f}=0,31 \mathrm{l} / \mathrm{s}\right)$.

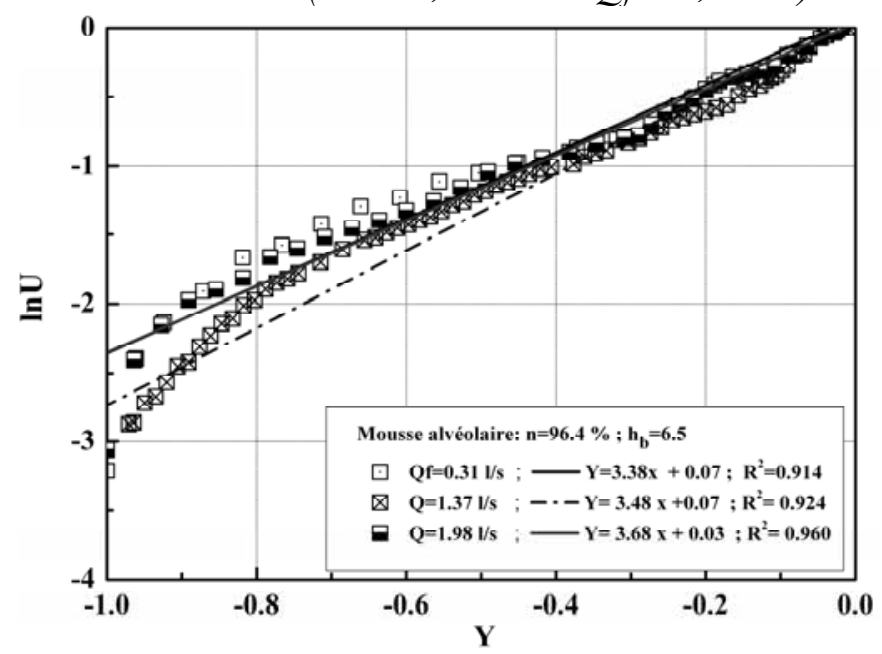

Figure 8(a). Evolution du paramètre $\alpha$ : cas des mousses alvéolaires.

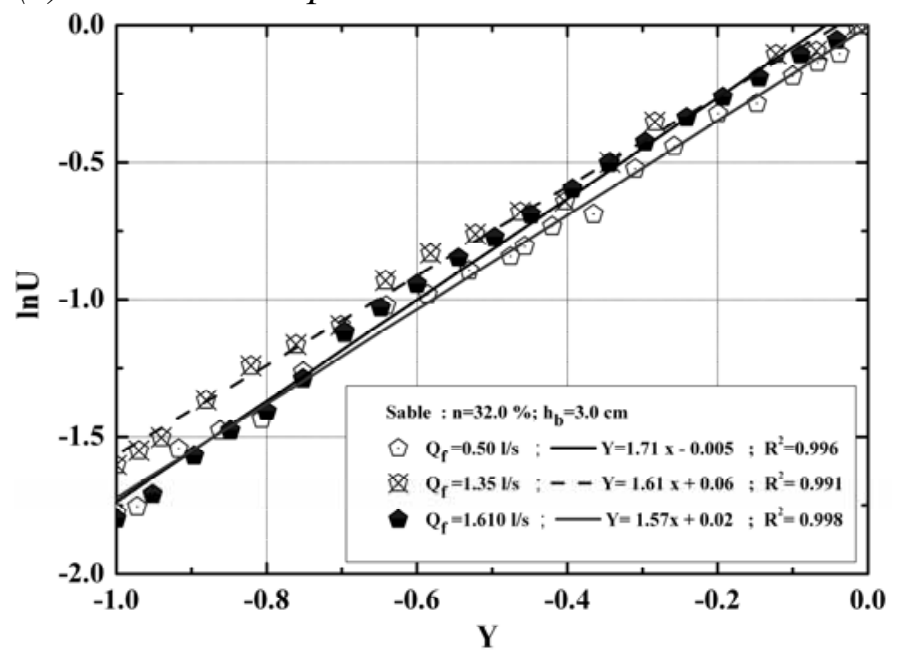

Figure 8(b). Evolution du paramètre $\alpha$ : cas du sable. 


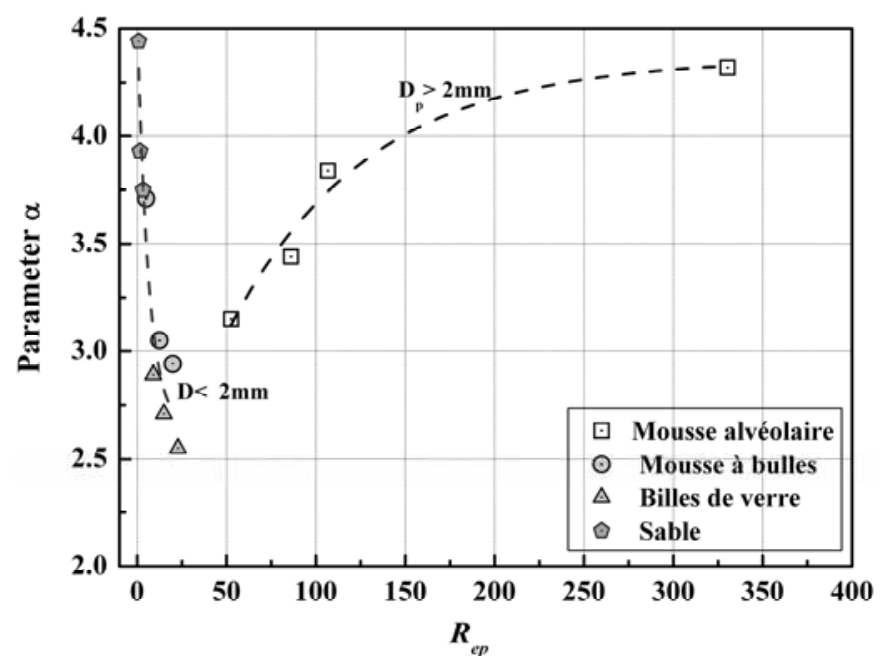

Figure 9. Variation du paramètre $\alpha$ en fonction du nombre de Reynolds et de la taille des pores.

\section{Conclusion}

Les résultats mis en évidence de mesures des vitesses par la technique de VDU au sein d'un écoulement à surface libre sur un lit perméable montrent que le profil des vitesses est non nul au sein du lit. L'écoulement en milieu poreux est de forme exponentielle qui se caractérise par un gradient de vitesse important à l'interface eau-sédiment.

Ceci permet de montrer que les modèles classiques de détermination de la contrainte pariétale et de frottement $\tau_{p}$ à la paroi ne sont pas bien adaptés car ils sont basés principalement sur les caractéristiques de l'écoulement libre sans prendre en compte la nature du sédiment, ses caractéristiques (porosité) et les caractéristiques de l'écoulement interstitiel (nombre de Reynolds des pores).

Cette contrainte de frottement $\tau_{p}$ est associée au seuil de mouvement. Il semble donc important de la définir en considérant, en plus des caractéristiques de l'écoulement libre, les caractéristiques du sédiment et surtout de l'écoulement interstitiel. Cet objectif est celui des travaux actuels qui consistent à étudier l'effet du cisaillement sous l'action notamment du phénomène du swash.

\section{Remerciements}

Les travaux ont été réalisés dans le cadre du Programme de Coopération intergouvernemental Franco-Algérien de formation supérieure (PROFAS). La collaboration de J-C Willemetz, directeur de Signal Processing SA a été très appréciée tout au long de l’étude, nous le remercions pour sa disponibilité. 
Étude de la répartition des vitesses interstitielles au sein d'un lit perméable sous un écoulement à surface libre : 1.13

\section{Références bibliographiques}

AHMED N., SUNADA D.K. (1969). Non linear flow in porous media. Journal of Hydraulic Division ASCE, Vol. 95, (HY6), pp 1847-1857.

CARPENTIER, G. (2006). Etude expérimentale des effets de perméabilité du soubassement des structures soumises à l'action de la houle. Thèse, Université de Caen, 216 p.

COMITI J., RENAUD M. (1989). A new model for determining mean structure parameters of fixed beds from pressure drop measurements: application to beds packed with parallelepipedal particles. Chemical Engineering Sciences, Vol. 44, pp 1539-1545.

GRAF W.H., ALTINAKAR M.S. (1995). Hydrodynamique. Presses Polytechniques et Universitaires Romandes, Lausanne, 481 p.

KLAR M. (2005). Design of an endoscopic 3-D particle-tracking velocimetry system and its application in flow measurements within a gravel layer. Thesis, university of Heidelberg, 278 p.

LHERMITTE R. (1983). Doppler sonar observation of tidal flow. Journal of Geophysical Research, Vol. 88, pp 725-742.

MICHAUX-LEBLOND N., BELORGEY M., ATTIACH J. (1996). Benard-von Karman vortex street development behind a heated cylinder. Proceeding of first International Symposium on Ultrasonic Doppler Methods for Fluid Mechanics and Fluid Engineering, Villigen Paul Scherrer Institute, Switzerland, pp 53-56.

MONTILLET A. (1995). Fiabilité de la détermination de paramètres structuraux de mousses synthétiques à partir des mesures de chute de pression. Récents progrès en Génie des Procédés, Vol. 9, pp 125-130.

SHIMIZU Y., TSUJIMOTO T., NAKAGAWA H. (1990). Experiment and macroscopic modelling of flow in highly permeable porous medium under freesurface flow. Journal of Hydroscience and Hydraulic Engineering, Vol. 8(1), pp 69-78.

WAHYUDI I. (1998). Ecoulement dans les sols granulaires saturés en régime permanent et transitoire. Thèse, Université de Nantes, 195 p.

WAHYUDI I., MONTILLET A., KAHLIFA A. (2002). Darcy and post-Darcy flows within different sands. Journal of Hydraulic Research, Vol. 40, pp 519525.

WARD J.C. (1964). Turbulent flow in porous Media. Journal of Hydraulic Division ASCE, Vol. 90, (HY5), pp 1-12.

WILLEMETZ J.C. (1990). Étude quantitative de l'hémodynamique de vaisseaux sanguins profonds par échographie Doppler ultrasonore. Thèse, Ecole Polytechnique Fédérale de Lausanne (EPFL), 158 p. 


\section{Nomenclature}

$a_{o}, b_{o}$ : coefficients de l'équation de Forchheimer

$(s / m),\left(s^{2} / m^{2}\right)$

$b$ : largeur du canal

$c$ : vitesse du son dans l'eau $(c \cong 1500 \mathrm{~m} / \mathrm{s})$

$f_{0}$ : fréquence d'émission de l'onde ultrasonore

$f_{D}$ : fréquence du Doppler

$h_{b}: \quad$ hauteur du lit de sédiments

$h_{w}$ : tirant d'eau de la veine fluide

$H: \quad$ tirant d'eau total, $h=h_{w}+h_{b}$

$i$ : gradient hydraulique

$n$ : porosité totale

$u$ : vitesse d'écoulement interstitielle

$(\mathrm{cm} / \mathrm{s})$

$D, D_{50}$ : diamètre, diamètre médian des particules

$Q_{f}$ : débit d'écoulement dans la veine fluide

$R_{h}$ : rayon hydraulique de la veine fluide.

$\alpha$ : paramètre expérimental caractérisant l'écoulement du milieu poreux.

$\theta: \quad$ angle du faisceau ultrasonore avec l'axe de l'écoulement.

$R e_{p}: \quad$ nombre de Reynolds des pores, $R_{e p}=\frac{\rho \cdot u \cdot D_{p}}{\mu(1-n)}$

$D_{p}: \quad$ diamètre hydraulique moyen des pores $D_{p}=\frac{2}{3} \cdot \frac{n \cdot D}{(1-n)}$

(mm)

$Y: \quad$ rapport de la position de la vitesse sur la hauteur du lit, $Y=\frac{z}{h_{b}}$

$U: \quad$ rapport de la vitesse sur la vitesse maximale interstitielle, $U=\frac{u}{u_{\max }}$

$V: \quad$ vitesse débitante de la veine fluide seule; $V=0,82 V_{\max }\left[\frac{\left(1+0,6 \sqrt{R_{h}}\right)}{\left(1+0,9 \sqrt{R_{h}}\right)}\right]$

Re : $\quad$ nombre de Reynolds de l'écoulement dans la veine fluide $; \mathrm{R}_{\mathrm{e}}=\frac{V \cdot R_{h}}{v}$

Fr: $\quad$ nombre de Froude de l'écoulement associé à la veine ; $F_{R}=\frac{V}{\sqrt{g h_{w}}}$ 